\title{
Real-time detection of state transitions in stochastic signals from biological systems
}

\author{
Max H. Bergkamp ${ }^{1,3}$, Leo J. van IJzendoorn ${ }^{2,3}$, Menno W.J. Prins ${ }^{1,2,3,4 *}$
}

${ }^{1}$ Department of Biomedical Engineering, Eindhoven University of Technology, The Netherlands 2Department of Applied Physics, Eindhoven University of Technology, The Netherlands ${ }^{3}$ Institute for Complex Molecular Systems (ICMS), Eindhoven University of Technology, The Netherlands

${ }^{4}$ Helia BioMonitoring, Eindhoven, The Netherlands

*Email: m.w.j.prins@tue.nl

\section{Supporting Information}

\section{Derivation of maximum-likelihood estimators}

We assume that the probability density function of a variable $x_{i}$ is given by:

$$
f\left(x_{i}, \mu, \sigma, \epsilon\right)=\sqrt{\frac{1}{2 \pi \sigma^{2}}} \exp \left(-\frac{\left(x_{i}-\epsilon x_{i-1}-(1-\epsilon) \mu\right)^{2}}{2 \sigma^{2}}\right)
$$

This represents a distribution with a mean $\mu$, standard deviation $\sigma$ and nearest-neighbor coupling parameter $\epsilon$ as model parameters.

The log-likelihood of the assumed distribution in a time trace of $N$ data points is given by:

$$
g\left(x_{i}, \mu, \sigma, \epsilon\right)=\log \left(\prod_{i=1}^{N} f\left(x_{i}, \mu, \sigma, \epsilon\right)\right)=\sum_{i=1}^{N} \log \left(f\left(x_{i}, \mu, \sigma, \epsilon\right)\right)
$$

Combining equations 1 and 2 gives:

$$
g\left(x_{i}, \mu, \sigma, \epsilon\right)=-\frac{N}{2} \log (2 \pi)-\frac{N}{2} \log \left(\sigma^{2}\right)-\sum_{i=1}^{N} \frac{\left(x_{i}-\epsilon x_{i-1}-(1-\epsilon) \mu\right)^{2}}{2 \sigma^{2}}
$$

By maximization of equation 3 we can obtain the maximum-likelihood estimators (MLE), $\hat{\mu}, \hat{\epsilon}$ and $\hat{\sigma}$. This is done by calculating the partial derivatives of $g\left(x_{i}, \mu, \sigma, \epsilon\right)$ with respect to each of the model parameters. Subsequently the maximum can be found by setting the partial derivative equal to zero.

\subsection{MLE nearest-neighbor coupling}

The partial derivative with respect to $\epsilon$ is given by:

$$
\frac{\partial g}{\partial \epsilon}=-\frac{1}{\sigma^{2}} \sum_{i=1}^{N}\left(\mu-x_{i-1}\right)\left(\epsilon\left(\mu-x_{i-1}\right)+x_{i}-\mu\right)
$$

To find an expression for $\hat{\epsilon}$ we need to set the partial derivative to zero, which gives:

$$
\hat{\epsilon}=\frac{N \mu^{2}+\sum_{i=1}^{N} x_{i} x_{i-1}-\mu\left(x_{i}+x_{i-1}\right)}{N \mu^{2}+\sum_{i=1}^{N} x_{i-1} x_{i-1}-2 \mu x_{i-1}}
$$


We need an estimation of $\mu$ to calculate $\hat{\epsilon}$. However, an estimation of $\epsilon$ is also needed to calculate $\hat{\mu}$ as we will show later. The MLEs for both parameters cannot be expressed independently. In order to avoid complex and computationally expensive calculations, we use the arithmetic mean to get an expression for $\hat{\epsilon}$ :

$$
\mu_{a}=\frac{1}{N} \sum_{i=1}^{N} x_{i}
$$

The MLE for the nearest-neighbor coupling parameter is then given by:

$$
\hat{\epsilon}=\frac{N \mu_{a}^{2}+\sum_{i=1}^{N} x_{i} x_{i-1}-\mu_{a}\left(x_{i}+x_{i-1}\right)}{N \mu_{a}^{2}+\sum_{i=1}^{N} x_{i-1} x_{i-1}-2 \mu_{a} x_{i-1}}
$$

\subsection{MLE mean}

The partial derivative with respect to $\mu$ is given by:

$$
\frac{\partial g}{\partial \mu}=-\frac{1}{2 \sigma^{2}} \sum_{i=1}^{N}-2(1-\epsilon)\left(x_{i}-\epsilon x_{i-1}-(1-\epsilon) \mu\right)
$$

This can be rewritten as:

$$
\frac{\partial g}{\partial \mu}=\frac{1-\epsilon}{\sigma^{2}}\left[-\mathrm{N}(1-\epsilon) \mu+\sum_{i=1}^{N} x_{i}-\epsilon x_{i-1}\right]
$$

To find an expression for $\hat{\mu}$ we need to set the partial derivative to zero, which gives:

$$
\hat{\mu}=\frac{1}{N(1-\epsilon)} \sum_{i=1}^{N} x_{i}-\epsilon x_{i-1}
$$

We will further simplify this equation by using the following expression:

$$
\sum_{i=1}^{N} \epsilon x_{i-1}=\epsilon\left(x_{0}-x_{n}+\sum_{i=1}^{N} x_{i}\right)
$$

Combining equations 10 and 11 gives:

$$
\hat{\mu}=\frac{\epsilon\left(x_{n}-x_{0}\right)}{N(1-\epsilon)}+\frac{1-\epsilon}{N(1-\epsilon)} \sum_{i=1}^{N} x_{i}
$$

We obtain the final expression for $\hat{\mu}$ by using the MLE for the nearest-neighbor coupling parameter:

$$
\hat{\mu}=\frac{1}{N}\left(\frac{\hat{\epsilon}\left(x_{n}-x_{0}\right)}{1-\hat{\epsilon}}+\sum_{i=1}^{N} x_{i}\right)
$$




\subsection{MLE variance}

The partial derivative with respect to $\sigma^{2}$ is given by:

$$
\frac{\partial g}{\partial \sigma^{2}}=-\frac{N}{2 \sigma^{2}}+\frac{1}{2 \sigma^{4}} \sum_{i=1}^{N}\left(x_{i}-\epsilon x_{i-1}-(1-\epsilon) \mu\right)^{2}
$$

To find an expression for $\hat{\sigma}^{2}$ we need to set the partial derivative to zero, which gives:

$$
\hat{\sigma}^{2}=\frac{1}{N} \sum_{i=1}^{N}\left(x_{i}-\hat{\epsilon} x_{i-1}-(1-\hat{\epsilon}) \hat{\mu}\right)^{2}
$$

\subsection{Expression for log-likelihood}

Finally, the log-likelihood can be calculated by plugging in the MLEs of the model parameters:

$$
g\left(x_{i}, \hat{\mu}, \hat{\sigma}, \hat{\epsilon}\right)=-\frac{N}{2} \log \left(2 \pi \hat{\sigma}^{2}\right)-\sum_{i=1}^{N} \frac{\left(x_{i}-\hat{\epsilon} x_{i-1}-(1-\hat{\epsilon}) \hat{\mu}\right)^{2}}{2 \hat{\sigma}^{2}}
$$

The second term can be simplified by using equation 15 :

$$
g\left(x_{i}, \hat{\mu}, \hat{\sigma}, \hat{\epsilon}\right)=\frac{N}{2} \log \left(\frac{1}{2 \pi \hat{\sigma}^{2}}\right)-\frac{N \hat{\sigma}^{2}}{2 \hat{\sigma}^{2}}
$$

Rewriting this expression gives:

$$
g\left(x_{i}, \hat{\mu}, \hat{\sigma}, \hat{\epsilon}\right)=\frac{N}{2}\left(\log \left(\frac{1}{2 \pi \hat{\sigma}^{2}}\right)-1\right)
$$

\subsection{Fast calculation of MLEs in windows of data points with cumulative sums}

The following cumulative sums are calculated as a function of data point index $n$ :

$$
\begin{gathered}
X(n)=\sum_{i=1}^{n} x_{i} \\
Y(n)=\sum_{i=1}^{n} x_{i} x_{i} \\
Z(n)=\sum_{i=2}^{n} x_{i} x_{i-1}
\end{gathered}
$$

These cumulative sums are calculated for all $n \leq N$. Additionally, we set these expressions to zero for certain values of $n$ :

$$
X(0)=Y(0)=Z(0)=Z(1)=0
$$


The cumulative sums in a window of data points that starts at $\mathrm{j}$ and ends at $\mathrm{k}$ can be calculated from the cumulative sums defined in equations (19-22):

$$
\begin{gathered}
X_{j, k}=\sum_{i=\mathrm{j}}^{k} x_{i}=\sum_{i=1}^{k} x_{i}-\sum_{i=1}^{j-1} x_{i}=X(k)-X(j-1) \\
Y_{j, k}=\sum_{i=\mathrm{j}}^{k} x_{i} x_{i}=Y(k)-Y(j-1) \\
Z_{j, k}=\sum_{i=\mathrm{j}}^{k} x_{i} x_{i-1}=Z(k)-Z(j-1)
\end{gathered}
$$

The number of data points in a window is given by:

$$
N_{w}=k-j+1
$$

The arithmetic mean of a window of data points that starts at $\mathrm{j}$ and ends at $\mathrm{k}$ is given by:

$$
\mu_{a}=\frac{X_{j k}}{N_{w}}
$$

With $\mu_{a}$ we can find an expression for $\hat{\epsilon}$ :

$$
\hat{\epsilon}=\frac{N_{w} \mu_{a}^{2}+Z_{j, k}-\mu_{a}\left(X_{j, k}+X_{j-1, k-1}\right)}{N_{w} \mu_{a}^{2}+Y_{j-1, k-1}-2 \mu_{a} X_{j-1, k-1}}
$$

The MLE for the mean can now be calculated by substituting the expression for $\hat{\epsilon}$ (eq 28) in equation 13:

$$
\hat{\mu}=\frac{1}{N_{w}}\left(\frac{\hat{\epsilon}\left(x_{k}-x_{j-1}\right)}{1-\hat{\epsilon}}+X_{j, k}\right)
$$

Finally, we can find an expression for $\hat{\sigma}^{2}$ in terms of the cumulative sums:

$$
\hat{\sigma}^{2}=\frac{1}{N_{w}}\left(Y_{j, k}-2 \hat{\epsilon}^{2} Y_{j-1, k-1}-\hat{\mu}^{2}(1-\hat{\epsilon})^{2}-2 \hat{\epsilon} Z_{j, k}-2 \hat{\mu}(1-\hat{\epsilon})\left(X_{j, k}-\hat{\epsilon} X_{j-1, k-1}\right)\right)
$$




\section{Biosensing by Particle Mobility (BPM)}

Experimental data of BPM experiments is characterized by a complex interplay between state properties and binding kinetics. The tethered particle moves according to Brownian motion, which causes time-correlation of data points within a state. The unbound state is geometrically defined by properties including the tether length, particle size and particle roughness, which limit the particle motion ${ }^{1}$. Bound states show even more variability in their geometrical confinements since they also depend on the location of the additional bond with respect to the tether. The switching frequency between bound and unbound states depends on properties such as binder molecule areal density on the particle and on the surface, target molecule concentration and the molecular affinities. Additionally, experimental parameters such as localization error, experimental drift and motion blur influence the measured particle positions. As a result, experimental position time traces of BPM can be regarded as complex and include multiple variable states with a wide range of lifetimes.

\subsection{BPM simulations}

The aim of the simulations is to evaluate the ability of the CPD algorithm to accurately detect changes in distributions that are typically observed in BPM. Therefore, the focus lies on simulating states with a wide variety in distributions and lifetimes. Including all the physical details of the BPM system in e.g. a Brownian dynamics simulation model, is beyond the scope of this research.

Instead we developed a two-dimensional simulation model for BPM. Data is generated by simulating free diffusion of a particle in a confined region that characterizes the state. A random displacement is generated in each dimension separately according to the probability density function for free diffusion²:

$$
P_{1 D}(\Delta x, \Delta t)=\frac{1}{\sqrt{4 \pi D \Delta t}} \exp \left(-\frac{\Delta x^{2}}{4 D \Delta t}\right)
$$

Each diffusion step is accepted or rejected according to a position dependent acceptance probability function. The unbound state is defined by a circular confined region with radius $R$. The acceptance probability of the unbound state is given by:

$$
P(x, y)=\left\{\begin{aligned}
1-\left(\frac{x^{2}+y^{2}}{R^{2}}\right), & x^{2}+y^{2}<R^{2} \\
0, & x^{2}+y^{2} \geq R^{2}
\end{aligned}\right.
$$

Bound states are defined by elliptical confined regions and are characterized by a center $\left(x_{c}, y_{c}\right)$, amplitudes $\left(x_{a m p}, y_{a m p}\right)$ and an angle $\theta$ between the $x$-axis and the axis of the ellipse along $x_{a m p}$. The acceptance probability of a bound state is given by:

$$
P(x, y)=\left\{\begin{aligned}
1-\left(\frac{\left(\left(x-x_{c}\right) \cos (\theta)+\left(y-y_{c}\right) \sin (\theta)\right)^{2}}{x_{a m p}^{2}}+\frac{\left(\left(x-x_{c}\right) \sin (\theta)+\left(y-y_{c}\right) \cos (\theta)\right)^{2}}{y_{a m p}^{2}}\right), & x^{2}+y^{2}<R^{2} \\
0, & x^{2}+y^{2} \geq R^{2}
\end{aligned}\right.
$$

Figure $\mathrm{S} 1$ shows the acceptance probabilities and simulated motion patterns for the unbound states and a single bound state. 

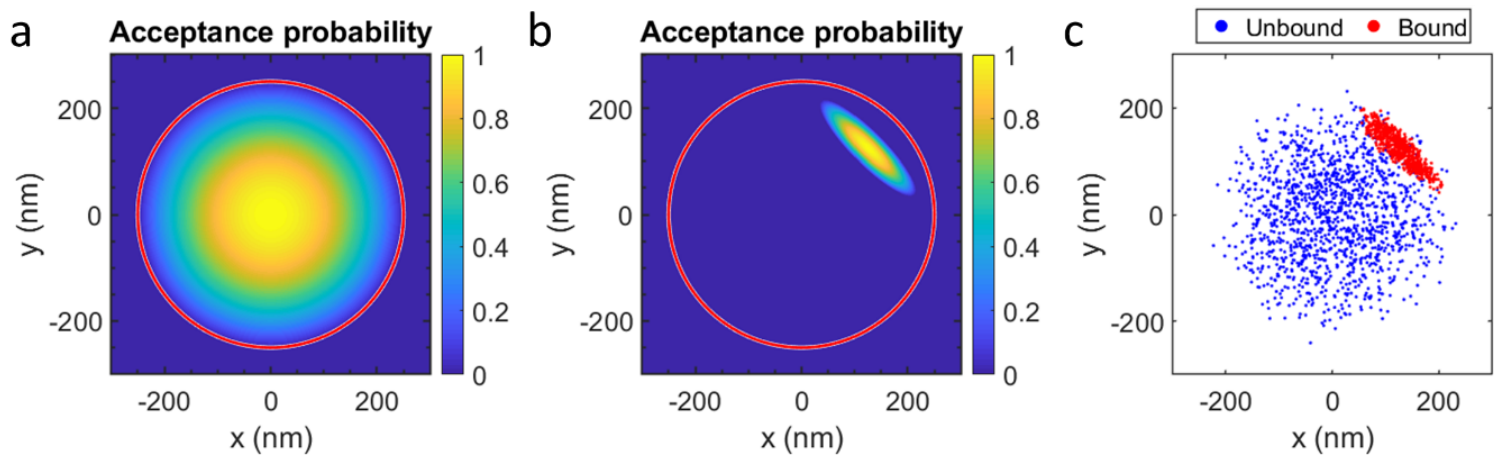

Figure S1: (a) Acceptance probability of the unbound state. (b) Acceptance probability of an elliptical bound state. (c) Motion pattern of simulated BPM data that includes both the unbound state (blue) and the bound state (red).

Switching between bound and unbound states is implemented by generating singleexponentially distributed lifetimes. The particle switches from the unbound state to the bound state after the generated lifetime has passed and the particle is within a confinement of a bound state. Switching from the bound state to the unbound state directly occurs after the generated lifetime has passed. The model includes measurement noise by adding white Gaussian noise to the simulated data. This model is representative for experimental BPM data, since it includes a wide range of state lifetimes, multiple variable state distributions, and a diffusion constant.

\subsection{BPM simulation settings}

Table S1 shows the settings that were chosen to perform the BPM simulations. The sampling rate was chosen equal to the typical value used in BPM experiments ${ }^{3-5}$. The theoretical bulk diffusion coefficient for a BPM particle with a radius of $500 \mathrm{~nm}$ in water at room temperature is: $D=4.4 \cdot 10^{-13} \mathrm{~m}^{2} \mathrm{~s}^{-1}$. The radius of the motion pattern in the unbound state was set to a typical value of $250 \mathrm{~nm}$. For each time trace, the acceptance probabilities of multiple bound states are randomly generated according to the specified ranges of the bound state center, amplitudes and angle that are provided in table S1.

Table S1: Simulation parameters for BPM

\begin{tabular}{|l|l|}
\hline Simulation parameter & Value \\
\hline Sampling rate & $30 \mathrm{~Hz}$ \\
\hline Diffusion constant $D$ & $4.4 \cdot 10^{-13} \mathrm{~m}^{2} \mathrm{~s}^{-1}$ \\
\hline Unbound state radius $R$ & $250 \mathrm{~nm}$ \\
\hline Number of randomly generated bound states & 20 \\
\hline Bound state center $\left(x_{c}, y_{c}\right)$ & $\left(0 \leq x_{c} \leq R\right),\left(0 \leq y_{c} \leq R\right),\left(x_{c}^{2}+y_{c}^{2} \leq R^{2}\right)$ \\
\hline Bound state amplitudes $\left(x_{a m p}, y_{a m p}\right)$ & $\left(0.1 R \leq x_{a m p} \leq 0.5 R\right),\left(0.1 R \leq y_{a m p} \leq 0.5 R\right)$ \\
\hline Bound state angle $\theta$ & $(0 \leq \theta<2 \pi)$ \\
\hline Lifetimes (single-exponentially distributed) & Variable \\
\hline Standard deviation of Gaussian noise & $5 \mathrm{~nm}$ \\
\hline
\end{tabular}




\section{Extracting state lifetimes}

Bound and unbound state lifetimes can be extracted from experimental BPM time traces with detected change points by classifying the states between consecutive change points.

In the first step, states are classified as unbound if the standard deviation in both $x$ - and $y$ direction is larger than $50 \mathrm{~nm}$ and smaller than $250 \mathrm{~nm}$. In the second step, states are classified as bound if the state is enclosed by two unbound states and the area of the state is smaller than the areas in the neighboring states. All other states are classified as undefined.

Figure S2 gives an example of an analyzed time trace with the classified states. The range between the first and fourth change point is classified as an undefined state. It is likely that the state between the second and third change point corresponds to a double-bound state, since the standard deviation is significantly smaller in this range. A more detailed analysis that includes classification of double-bound states is beyond the scope of this research.

For the unbound states, only the lifetimes are considered that are complete. In other words, unbound states at the boundaries of the trace are not considered. This might lead to an underestimation of the mean unbound state lifetime, since long unbound states are more likely to have overlap with the boundaries of the time trace.

For both bound and unbound states, false positives could lead to an underestimation of the mean state lifetimes. On the other hand, missing short-lived states might lead to an overestimation of the mean state lifetimes. In addition, non-specific binding or double-bound states might influence the lifetimes that are determined.

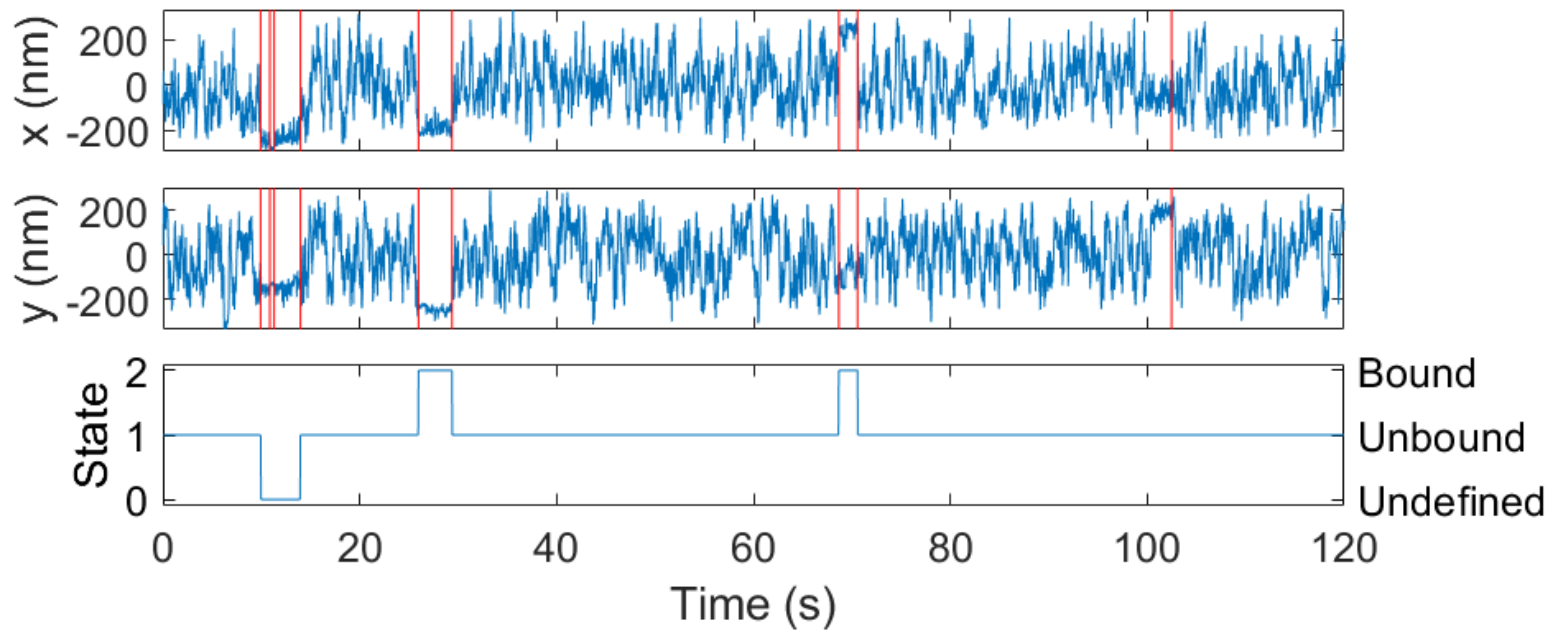

Figure S2: BPM $x$ and $y$ time traces with detected change points (red vertical lines). The bottom time trace shows the state time trace that is determined by classifying the states between consecutive change points. 


\section{Additional results simulated data}

\subsection{Nearest-neighbor coupling parameter}

The MM-CPD is designed to perform robust CPD in traces with varying time-correlated properties. This is achieved by including the nearest-neighbor coupling parameter $\epsilon$ in the distribution. By setting $\epsilon$ to zero, the assumed distribution becomes a Gaussian distribution (Equation 1). Figure S3a shows the effect of including $\epsilon$ in the distribution on the F1-score for a range of simulated diffusion constants with a reference diffusion constant $D_{0}=4.4 \cdot 10^{-13}$ $\mathrm{m}^{2} \mathrm{~s}^{-1}$ (i.e. to simulate the effect of particle size in a BPM system). The settings in table S1 were used for the simulations with mean bound and unbound state lifetimes of 2 and 20 seconds, respectively. Figure S3a shows a significant decrease of the F1-score for lower diffusion coefficients, especially for a Gaussian distribution $(\epsilon=0)$. Lower diffusion coefficients can lead to larger changes in mean and standard deviation between neighboring windows. Since a Gaussian distribution only includes a mean and standard deviation, the number of false positives increases for lower diffusion coefficients (Figure S3b). Including $\epsilon$ in the distribution significantly reduces the number of false positives that originate from diffusion effects.
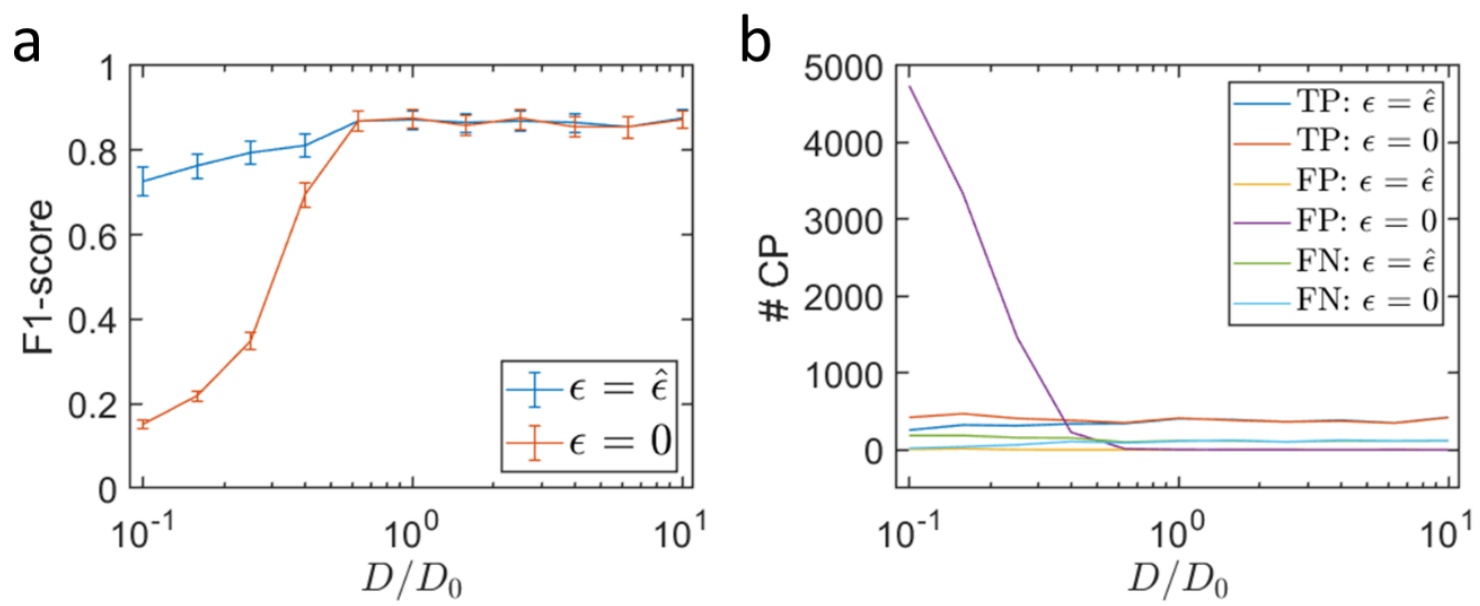

Figure S3: Influence of the nearest-neighbor coupling parameter $\epsilon$ on the performance of MMCPD. (a) F1-score as a function of the diffusion constant for a distribution with nearest-neighbor coupling parameter $(\epsilon=\hat{\epsilon})$ and a Gaussian distribution $(\epsilon=0)$. (b) Number of classified CPs: true positives (TP), false positives (FP) and false negatives (FN), as a function of the diffusion constant.

\subsection{CPU time as a function of state lifetime}

Figure S4 shows the CPU time as a function of the simulated average state lifetimes for the MM-CPD, IB-CPD and SMD-CPD. The MM-CPD algorithm shows a fast CPU time that is independent on the state lifetimes. The CPU time of the IB-CPD is clearly dependent on the state lifetimes, which is undesirable for real-time applications. The state dependent CPU time can be explained by the segmentation algorithm that is applied in IB-CPD. For time traces with shorter lifetimes the number of segmentations increases, which leads to the increased CPU time. 


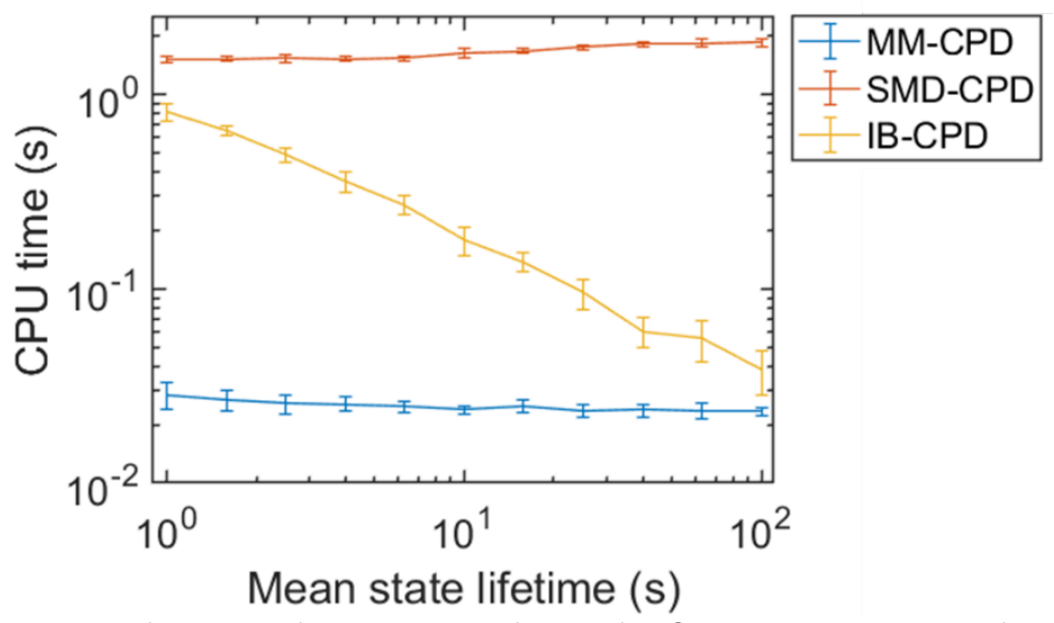

Figure S4: CPU time as a function of mean state lifetime for CPD in a time trace of 10.000 data points with a sampling rate of $30 \mathrm{~Hz}$.

\section{Additional results experimental data}

\subsection{State lifetime distributions}

Figure S5 shows the bound and unbound state lifetime distributions for different individual concentrations of analyte. About $90 \%$ of the detected bound states follow a single-exponential distribution for each analyte concentration. The average bound state lifetime shows no dependence on the concentration, which is expected, since the dissociation of the BPM particle is dependent on the interaction between the analogue and the detection molecules on the surface. The remaining $10 \%$ of the bound state lifetimes show slightly larger lifetimes, which can be attributed to multiple interactions or nonspecific binding ${ }^{4}$. The deviation from a singleexponential distribution is seen more clearly for higher analyte concentrations. This is as expected, since the number of specific interactions decreases as a function of analyte concentration. The unbound state lifetimes show multi-exponential distributions, which is caused by particle-to-particle variations in binder density ${ }^{6}$. Furthermore, the average unbound state lifetime increases as a function of analyte concentrations. This is as expected, since the analyte molecules block the formation of a bond between the analogue molecules on the particle and the detection molecules on the surface.
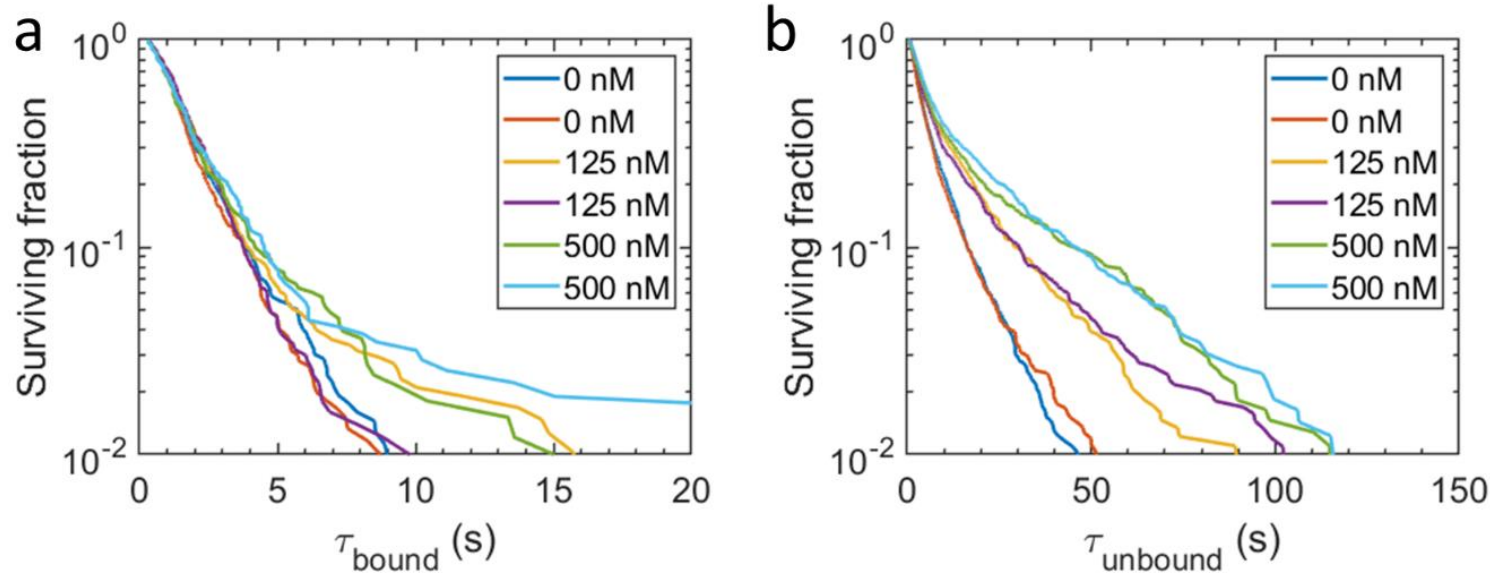

Figure S5: State lifetime distributions obtained with the MM-CPD algorithm. (a) Survival curves of the bound state lifetimes for different analyte concentrations. (b) Survival curves of the unbound state lifetimes for different analyte concentrations. 


\subsection{Threshold}

Figure S6 shows that the threshold can influence the values of the extracted physical parameters. The activities and bound state lifetime distributions are plotted before and after activation of the BPM system. Activation refers to the process of binding analogue molecules to the tethered particles. Before activation, no analogue molecules are present on the particles. Therefore, no change points and bound states are expected. However, Figure

S6a-c show that most particles show an activity larger than zero, especially with low threshold values. Figure S6d-f shows that also a distribution of bound state lifetimes is obtained before activation. These detected bound states could correspond to non-specific interactions, binding of an additional tether or false positives. After activation, the activity increases for all threshold values, indicating that the reversible binding between the analogue on the particle and detection molecules on the surface is measured. For lower thresholds, it is likely that a significant fraction of the detected bound states corresponds to non-specific interactions or false positives. Therefore, the measured bound state lifetime distribution will likely deviate from the true distribution. Additionally, it should be considered that the sensitivity for detecting shortlived states decreases as a function of the threshold. In summary, the measured bound state lifetime distribution is influenced both by missing short-lived states and by detection of false positive states. Increasing the threshold level leads to a smaller fraction of false positives, but a larger fraction of missed short-lived states. A threshold of 15 is probably too low, due to the high fraction of false positives. On the other hand, a threshold of 25 will likely result in an overestimation of the average bound state lifetime, due to the missing of short-lived states. Therefore, a threshold of 20 is considered as a good choice based on the quantitative evaluation with experimental data.
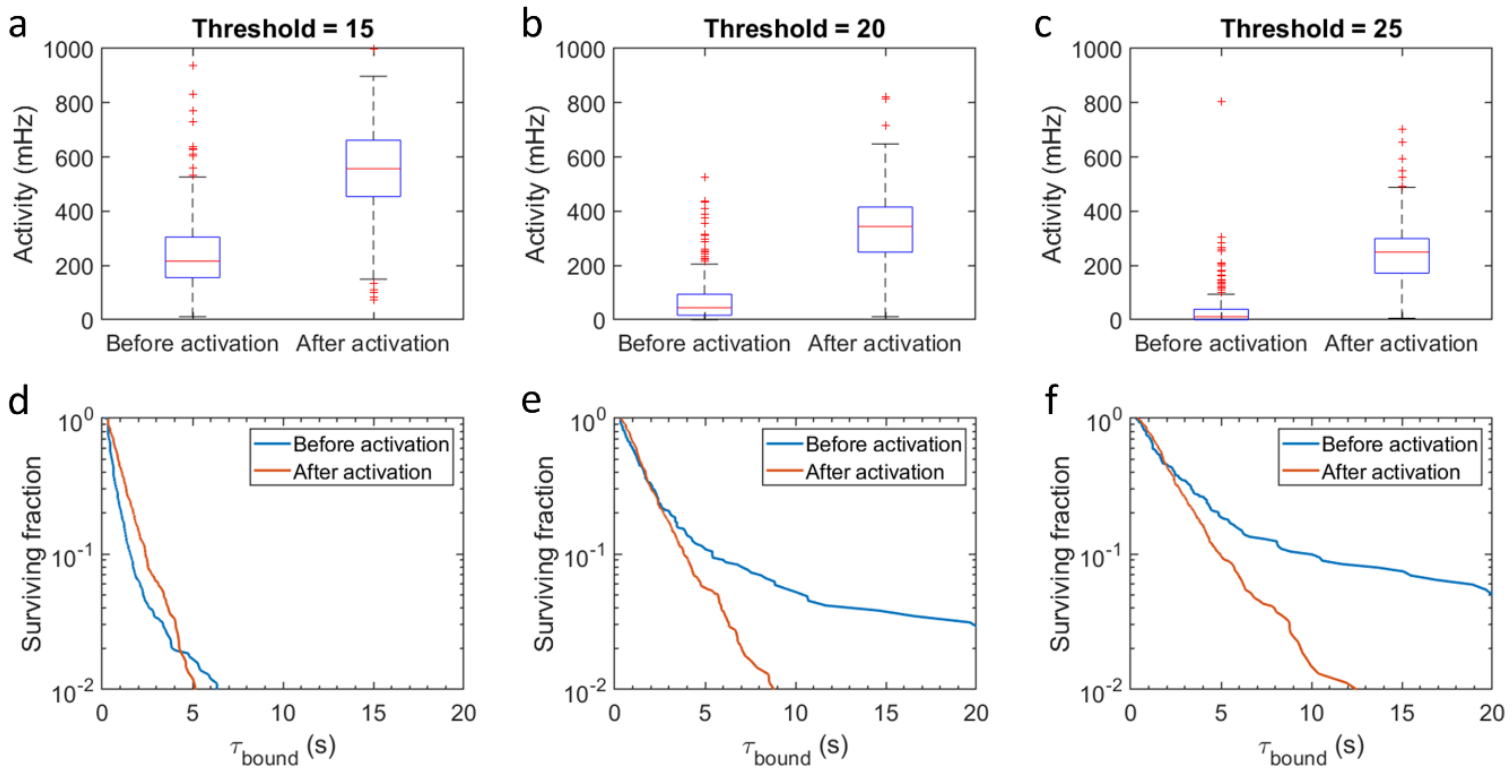

Figure S6: Influence of the threshold on the extracted physical parameters. (a)-(c) Box plots of the activities per particle before and after activation, i.e. functionalization of analogue molecules on the particle. The activities were calculated with three different thresholds. (d)-(f) Survival plots of the extracted bound state lifetimes before and after activation. 


\section{CPD methods from literature}

Table S2 gives an overview of methods that are frequently applied for CPD in biological applications. Most of these methods have also been applied to tethered particle motion data. Half-amplitude thresholding ${ }^{7}$ and hidden Markov models ${ }^{8}$ rely on prior knowledge of the available states, so these methods are not suited for analyzing systems with states that are not a priori known. Maximum-likelihood based methods ${ }^{9,10}$ can be applied without assumptions about underlying kinetic models or probability distributions. However, a maximum-likelihood approach based on a Poisson or Gaussian distribution is not robust for time traces that include states with time-correlated data points. More recently, an information-based maximumlikelihood CPD was introduced (IB-CPD) ${ }^{11}$. The physical model of this method includes a nearest-neighbor coupling parameter, allowing robust change point detection for timecorrelated data. The IB-CPD method is widely applicable for biophysical systems.

Table S2: Overview of algorithms for change point detection in time traces from biological applications.

\begin{tabular}{|c|c|c|c|c|c|}
\hline CPD Method & References & $\begin{array}{l}\text { A priori } \\
\text { undefined } \\
\text { states }\end{array}$ & $\begin{array}{l}\text { Robust for } \\
\text { time- } \\
\text { correlated } \\
\text { states }\end{array}$ & $\begin{array}{l}\text { Wide range } \\
\text { of state } \\
\text { lifetimes }\end{array}$ & $\begin{array}{l}\text { Computational } \\
\text { efficiency }\end{array}$ \\
\hline $\begin{array}{l}\text { Half-amplitude } \\
\text { thresholding } \\
\text { (HAT) }\end{array}$ & 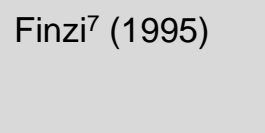 & No & No & No & Fast \\
\hline $\begin{array}{l}\text { Hidden Markov } \\
\text { models }\end{array}$ & $\begin{array}{l}\text { Beausang } \\
\text { (2007), } \\
\text { Johnson }^{12} \\
(2014)\end{array}$ & No & Yes & Yes & Slow \\
\hline $\begin{array}{l}\text { Maximum- } \\
\text { likelihood }\end{array}$ & $\begin{array}{l}\text { Watkins }^{9} \\
(2005), \\
\text { Manzo } \\
\text { (2010) }\end{array}$ & Yes & No & Yes & $\begin{array}{l}\text { Dependent on } \\
\text { lifetimes in time } \\
\text { trace } \\
\text { (segmentation) }\end{array}$ \\
\hline $\begin{array}{l}\text { Information- } \\
\text { based (IB-CPD) }\end{array}$ & $\begin{array}{l}\text { Wiggins }{ }^{11} \\
\text { (2015) }\end{array}$ & Yes & Yes & Yes & $\begin{array}{l}\text { Dependent on } \\
\text { lifetimes in time } \\
\text { trace } \\
\text { (segmentation) }\end{array}$ \\
\hline $\begin{array}{l}\text { Maximum- } \\
\text { likelihood } \\
\text { Multiple windows } \\
\text { (MM-CPD) }\end{array}$ & This paper & Yes & Yes & Yes & Fast \\
\hline
\end{tabular}




\section{References}

(1) Visser, E. W. A.; van IJzendoorn, L. J.; Prins, M. W. J. Particle Motion Analysis Reveals Nanoscale Bond Characteristics and Enhances Dynamic Range for Biosensing. ACS Nano 2016, 10 (3), 3093-3101. https://doi.org/10.1021/acsnano.5b07021.

(2) Chubynsky, M. V; Slater, G. W. Diffusing Diffusivity: A Model for Anomalous, yet Brownian, Diffusion. Phys. Rev. Lett. 2014, 113 (9), 098302. https://doi.org/10.1103/PhysRevLett.113.098302.

(3) Visser, E. W. A.; Yan, J.; van IJzendoorn, L. J.; Prins, M. W. J. Continuous Biomarker Monitoring by Particle Mobility Sensing with Single Molecule Resolution. Nat. Commun. 2018, 9 (1), 2541. https://doi.org/10.1038/s41467-018-04802-8.

(4) Yan, J.; van Smeden, L.; Merkx, M.; Zijlstra, P.; Prins, M. W. J. Continuous SmallMolecule Monitoring with a Digital Single-Particle Switch. ACS Sensors 2020, 5 (4), 1168-1176. https://doi.org/10.1021/acssensors.0c00220.

(5) Lubken, R. M.; de Jong, A. M.; Prins, M. W. J. Multiplexed Continuous Biosensing by Single-Molecule Encoded Nanoswitches. Nano Lett. 2020, 20 (4), 2296-2302. https://doi.org/10.1021/acs.nanolett.9b04561.

(6) Lubken, R. M.; de Jong, A. M.; Prins, M. W. J. How Reactivity Variability of Biofunctionalized Particles Is Determined by Superpositional Heterogeneities. ACS Nano 2021, 15 (1), 1331-1341. https://doi.org/10.1021/acsnano.0c08578.

(7) Finzi, L.; Gelles, J. Measurement of Lactose Repressor-Mediated Loop Formation and Breakdown in Single DNA Molecules. Science (80-. ). 1995, 267 (5196), 378-380. https://doi.org/10.1126/science.7824935.

(8) Beausang, J. F.; Nelson, P. C. Diffusive Hidden Markov Model Characterization of DNA Looping Dynamics in Tethered Particle Experiments. Phys. Biol. 2007, 4 (3), 205-219. https://doi.org/10.1088/1478-3975/4/3/007.

(9) Watkins, L. P.; Yang, H. Detection of Intensity Change Points in Time-Resolved Single-Molecule Measurements. J. Phys. Chem. B 2005, 109 (1), 617-628. https://doi.org/10.1021/jp0467548.

(10) Manzo, C.; Finzi, L. Quantitative Analysis of DNA-Looping Kinetics from Tethered Particle Motion Experiments. In Methods in Enzymology; 2010; pp 199-220. https://doi.org/10.1016/S0076-6879(10)75009-6.

(11) Wiggins, P. A. An Information-Based Approach to Change-Point Analysis with Applications to Biophysics and Cell Biology. Biophys. J. 2015, 109 (2), 346-354. https://doi.org/10.1016/j.bpj.2015.05.038.

(12) Johnson, S.; van de Meent, J.-W.; Phillips, R.; Wiggins, C. H.; Lindén, M. Multiple Lacl-Mediated Loops Revealed by Bayesian Statistics and Tethered Particle Motion. Nucleic Acids Res. 2014, 42 (16), 10265-10277. https://doi.org/10.1093/nar/gku563. 